\title{
Research on the Network Traversal based on Spatial Influence
}

Dpen Access

\author{
Ma Jun*, Wan Jie and Wang Wie \\ School of Safety \& Environmental Engineering, Capital University of Economics \& Business, Beijing, 100070, P.R. \\ China
}

\begin{abstract}
Different from other types of social networks, close social network have its own topologic features and propagation principles, and it is very common in social life, so it is worth research in depth. Student's network is a typical close network and suitable as a research object. The traversal of social network has been an important subject of social network analysis, and whether spatial factor is influencing factor need to verify. The importance of the topology characteristic value in student's network traversal process is analyzed. Finally, the concept of vitality is put forward to illustrate spatial influence of traversal based on high close student's network.
\end{abstract}

Keywords: High close network, spatial influence, topologic features, traversal closeness.

\section{INTRODUCTION}

Social network refers to the relationship collection formed by the actors in the society. A social network is composed of multiple nodes (representing the social actors) and collection composed of the directed or undirected attachment between each node (representing the relationship between actors) [1]. Interaction between individual members forms a relatively stable relationship of system, social network focus on the interaction between people and the contact, so social interaction will influence people's social behavior, and this interaction include friends, school mates, business partner, racial beliefs and so on. Social network analysis has many unique aspects compared with traditional social analysis method, but the most unique one is its research perspective. It focuses on research of the formation mechanism of the social structure and presented some social phenomenon from the perspective of social relations. The social structure also shows its diversity, economic or political structure, maybe behavior structure [2].

Most of the traditional social statistical method take individual as an independent unit to make statistical analysis through previous certain assumption that a person is considered as individual in the society. However, lacking of consideration about the real social mutual influence, the result analysis are one-sided. Limited by the limitations of theoretical knowledge and practical experience, the result was acceptable at that time. But with the rapid development of modern society and more complex relationships, traditional social research methods already cannot satisfy the requirement of scientific research [3].

Social network concluded that interaction between actors can't be independent. Interaction between researches should be considered before social network take the "relationship" as the unit, and structure viewed as relationship architecture between the actors change the rule and the internal structure mechanism based on relational data analysis of the social network structure (rather than the traditional social attribute data), and it is in turn mining analysis relationship analysis between the essence of the social phenomenon [4].

Patent WO058971, titled "Authenticated encryption support in iso/iec" [5], Patent WO056174, titled "Power saving in cellular networks" [6], Patent WO056650, titled "Altruistic scheduling in heterogeneous wireless networks" [7], Patent WO057610, titled "Communication apparatus" [8], Patent WO058842, titled "Distributed storage systems and methods" [9], Patent WO058379, titled "Communication apparatus" [10], Patent WO058842, titled "Nodes, systems and methods in a cellular network", invent a method of structuring network respectively. This method reveals the inherent relationship of network. Campus network is a special social relation network for its mass, reliable empirical data acquisition. Studying the relationship between campus students network can effectively explained some universal phenomena of the social network, dig deeper into the campus students with the mathematical relationship between the social network at the same time, and extended to various types of small group, for example, make quantitative analysis, implemental measures and evaluation on issues such as learning attitude influence each other in the education of primary and middle school students of the same class, fighting actively around all kinds of inner enterprise or other organizations, spread positive energy and so on .

\section{NETWORK BASIC CONCEPT}

\subsection{Network}

Based on graph theory, a network $\mathrm{G}$ can be expressed as a simple compose of nodes and edges, denoted as

$$
G=(\mathrm{V}(\mathrm{G}), \mathrm{E}(\mathrm{G}))
$$


$V(G)$ and $E(G)$ are the point set of network and the edge set respectively. An edge used to connect the node $i$ and $j(i, j \in \mathrm{V}(\mathrm{G}))$ can be called $(i, j)$ or $(j, i)$. Given $\mathrm{N}$ nodes of network $\mathrm{G}$, it can be only expressed as a adjacency matrix $\mathrm{A}[11]$ :

$A=\left(a_{i j}\right)_{N \times N}$,

where

$$
a_{\mathrm{ij}}= \begin{cases}1 & \text { Edge between node } i \text { and } j \\ 0 & \text { No edge between node } i \text { and } j\end{cases}
$$

For the weighted network, the adjacency matrix element represents the corresponding weight of the edge, and the weight usually reflects the measure of relationship strength. The matrix A is symmetric for undirected networks, excluding the self loops or multiple edges condition, and the diagonal element is 0 . If the network is directed, it cannot guarantee symmetry adjacency matrix. If adjacency matrix $a_{i j}=1$, node $i$ and node $j$ are adjacent. For node $i$, the degree of its is $k_{i}=\sum_{j} a_{i j}, k_{i}$ means the number of edges between $i$ and other nodes in network. The points set associated with the node $i$ called as $\Gamma(i)$. For weighted network, the degree of node is sum of weights of edges, which is also called the intensity of node.

\subsection{Path}

Given a network G, If two non-adjacent nodes can be connected from node $\mathrm{i}$ to node $\mathrm{j}$ through a sequence containing $m$ edges $\left(\left(i, s_{1}\right),\left(s_{1}, s_{2}\right), \cdots,\left(s_{m-1}, j\right) \in \mathrm{E}(\mathrm{G})\right), m$ edges are not repeated and the corresponding nodes also dose not repeated, then the set of $m$ edges is called as a path between $i$ and $j, m$ is the length of the path. The set of edges is a path between $i$ and $j$, where $n$ is the length of path. In general, there are many paths between $i$ and $j$. The shortest length of path is called as the shortest path. The length is called as the distance, and it is denoted as $d_{i j}$. For directed network, there is a path of length $n$ between $i$ and $j$ if the $n$ edges in the sequence $\left(\left(i, s_{1}\right),\left(s_{1}, s_{2}\right), \cdots,\left(s_{m-1}, j\right) \in \mathrm{E}(\mathrm{G})\right)$ of each edge have the same direction for directed network. There is a path of length $n$ between $i$ and $j$ if there are different direction of edges. The length of path is the sum of $n$ edge weights.

\subsection{Average Path Length [12]}

As defined above, for an undirected and unweighted network, the distance between $i$ and $j$ in network is defined as the shortest path edges connecting two nodes. Thus it extends a definition of describing the average path length of overall properties of network, the average distance between any two nodes in the network is the average path length of the network, which can be expressed as:

$$
L=\frac{1}{\frac{1}{2} N(N+1)} \sum_{i \geq j} d_{i j}
$$

where $\mathrm{N}$ is the number of network nodes.

\subsection{Degree and Degree Distribution}

The degree $k_{i}$ of node $i$ is the number of edges connecting directly to node $i$. The average degree of all nodes is called the average degree of network, denoted as $k_{i}$. Degree of a node is divided into out-degree and in-degree in directed network. The out-degree of a node means the number of edges from the node pointing to other nodes connecting with the node, while the in-degree of a node means the number of edges pointing to the node connecting with other nodes. Distribution of nodes in the network represents a distribution function, showing the probability of selecting an arbitrary node of degree $k$, which is the number of nodes of degree $k$ in the network accounts for the proportion of the total number of network nodes.

\subsection{Degree Centrality [13]}

Degree centrality is the definition of centricity in the basis of degree, considering the centrality of nodes is expressed by the degree of nodes. The expression of degree centrality can be expressed as:

$$
D C_{i}=\sum_{j \in \Gamma(i)} A_{i j}
$$

Degree centrality indicates the intensity of the node directly connecting with other nodes in the network. The greater intensity, the stronger importance it is. It is often used to simply measure the most important central figure of organization. People's position with highly centrality should be important in the organization.

\section{ESTABLISHMENT OF THE NETWORK AND TOPOLOGY ANALYSIS}

\subsection{Establishment of the Network}

The object of study is the whole students from an undergraduate class who study at night in the classroom, with the absence of a teacher or others intervention. Because some people are less determined, this leads to these students transform learning state into state without learning, which must affects the other students, accordingly, this transmission will have a bad effect between students. And in the absence of outside intervention, the impact will increase. At last, all the students are subject to the adverse effect.

The research object is 36 students from the same class, some of who live in the same dorm room. So they are relatively intimate in spite of a minor difference in the degree of intimacy. The closeness between the students is showed by the student network corresponding values in the adjacency matrix representation, and this closeness is various due to personal cognitive differences and individual differences, so students adjacency matrix corresponds to directed weighted network, the greater the value of the matrix is, the more intimate the relationship between two students is. Line values in the matrix indicate that the line corresponding to the students to other students' intimacy. Intimacy values obtained from the survey are divided into five grades $0-4$, the greater the value is, and the more intimate the relationship is. The basic flow is shown in Fig. (1). 


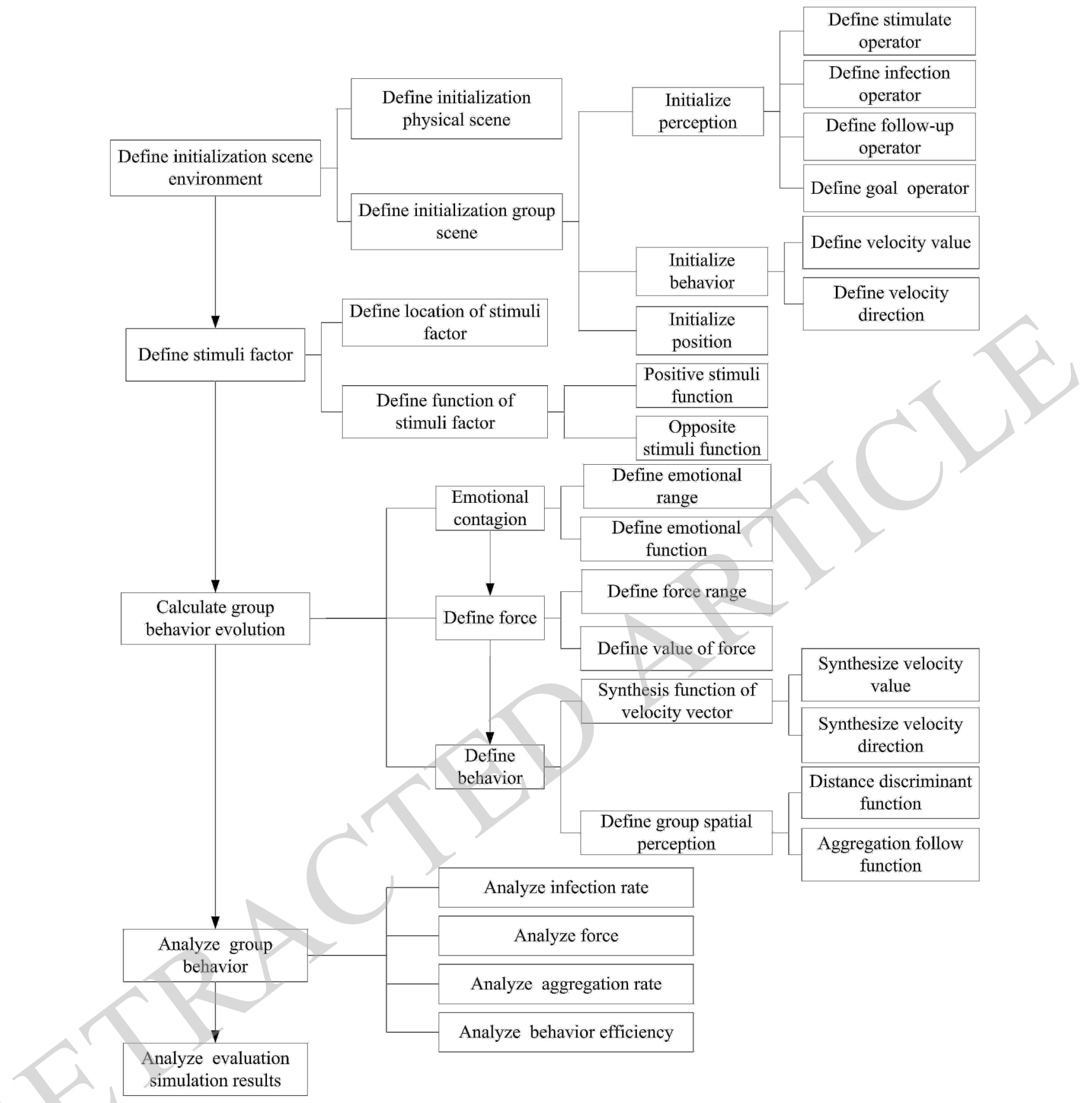

Fig. (1). The data processing.

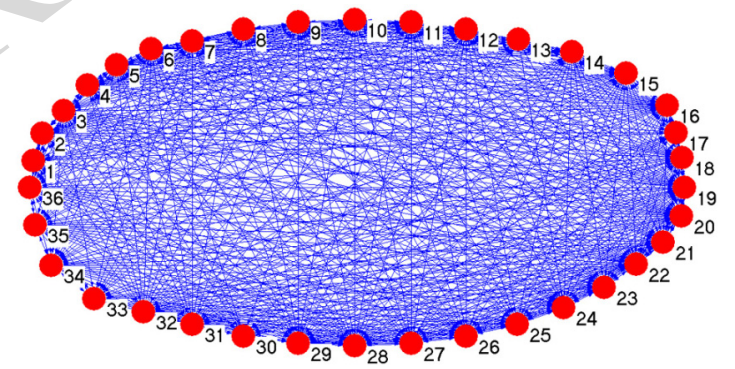

Fig. (2). The node distribution in the oval.

\subsection{Topology Analysis}

Apply the adjacency matrix of Pajek software to process to get two different node position of weighted directed graph.

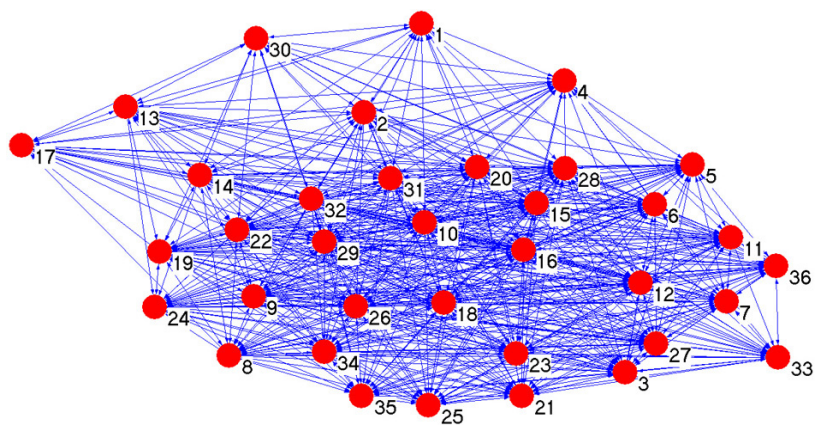

Fig. (3). The node distribution is randomness.

Clear from the above (Figs. $\mathbf{2}$ and $\mathbf{3}$ ), the student's network is a uniform network, its degree of each node is simi- 


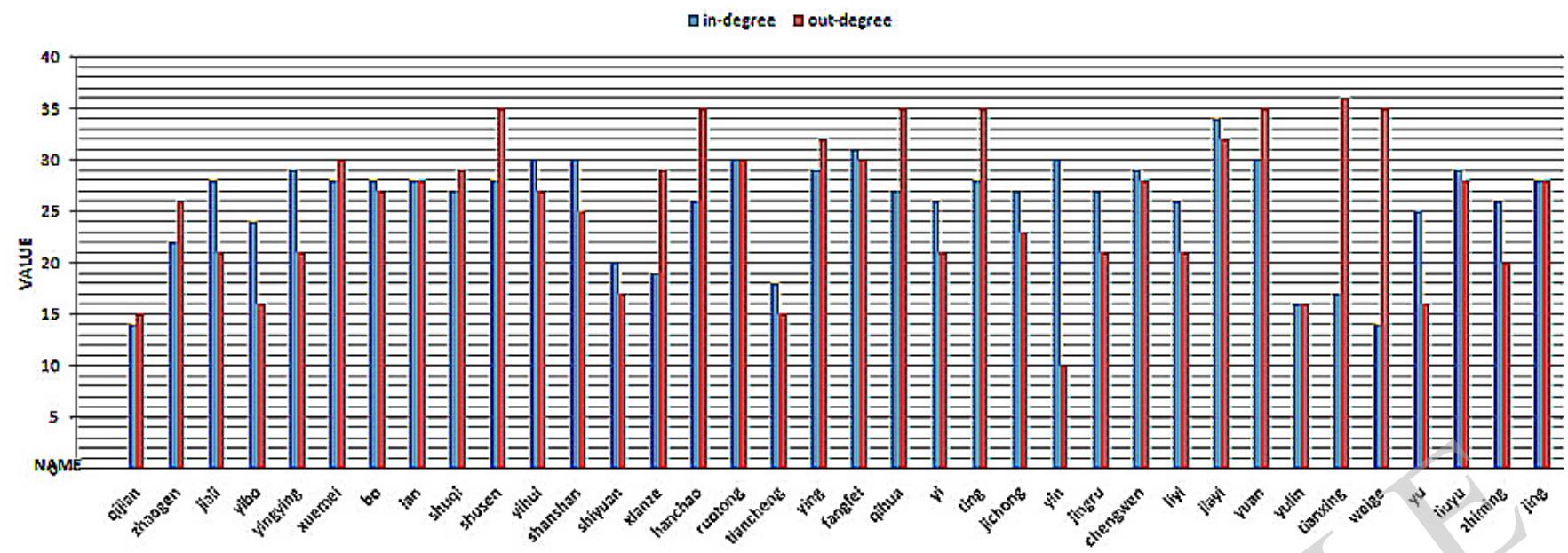

Fig. (4). The comparison chart of input and output degree.

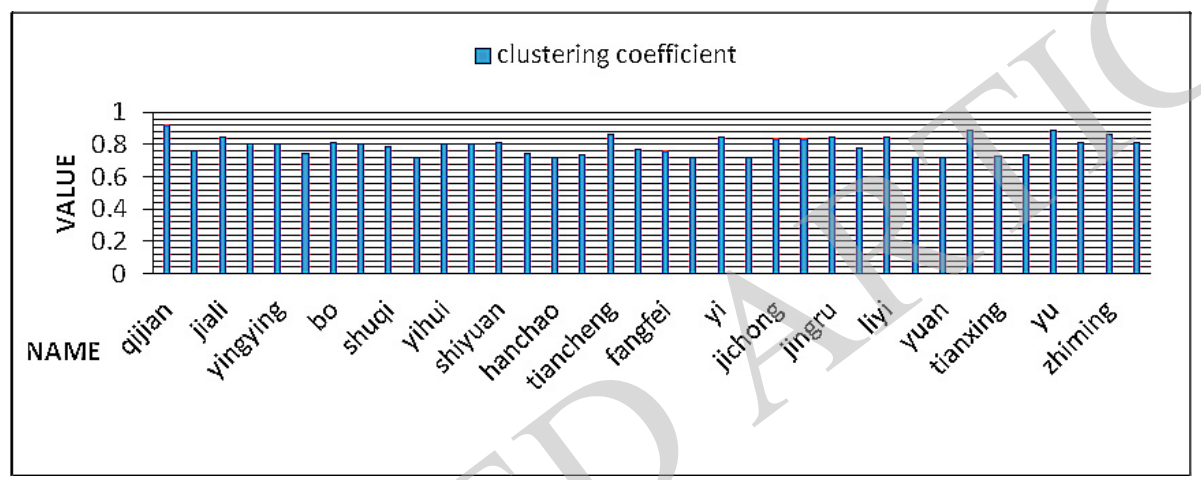

Fig. (5). The comparison chart of input and output degree.

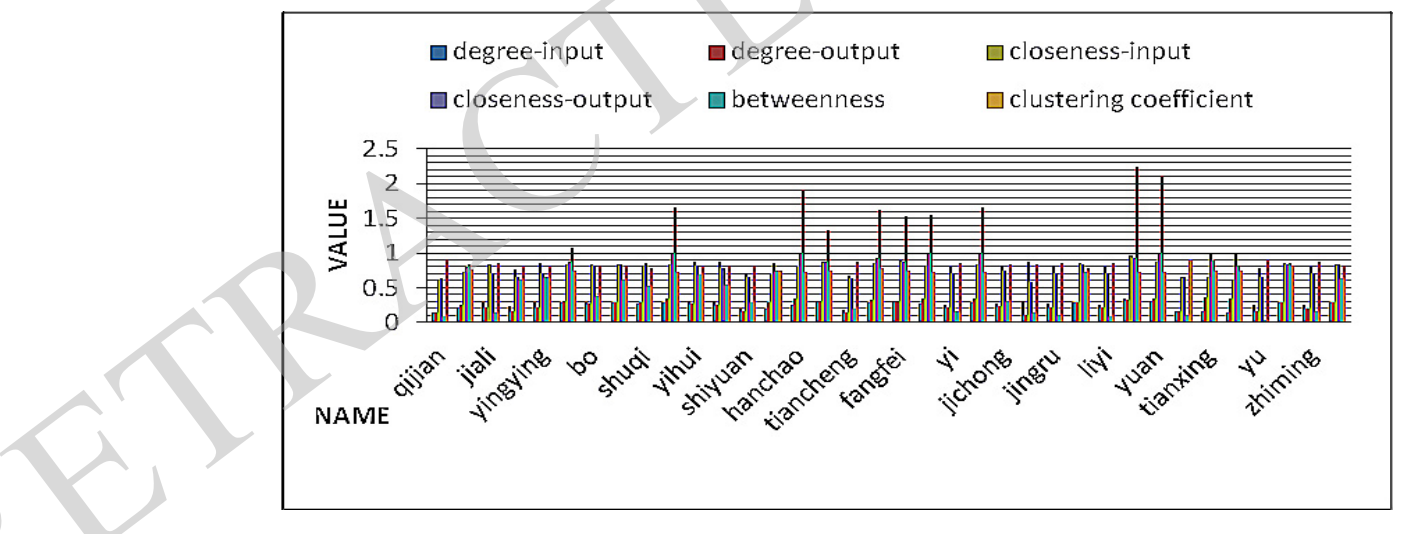

Fig. (6). The comprehensive comparison bar chart of topological property.

lar, because they are classmates, more daily communication. Assume the student's network is undirected, unweighted network, the average path length is 1.26429 , the diameter of 2 , and network average clustering coefficient is 0.79836 . These data show that the students network is a high intense, also from the above (Figs. 1 and 2). High density network is characterized by relatively important nodes in the network, but due to their relations are close, importance is not different too much. Histogram fully reflects the correlation of characteristic values. As observed in Fig. (4), the input and output degree of each node is similar, but some differences because of the cognitive between people. For example, I think you and I close degrees to 4 , and what do you think of our intimacy to 2 .
Line chart fully reflect the variation tendency of characteristic values. As observed in Figs. (4-6), the student network of all kinds of digital characteristic curve is very smooth, the similar, but the betweenness centrality curve are very volatile, it embodies the differences of students communicative competence, some students are good at communication, some not. The curve peak and the second highest node classmates corresponding to the men and women monitor in the class, as the dotted line in the Fig. (7), is also verifying the analysis. The other types of networks like BA network in the propagation characteristics are very different because of these dense student network characteristics, and the spread is rapid and devastating, so transmission characteristic of such network is analyzed. 


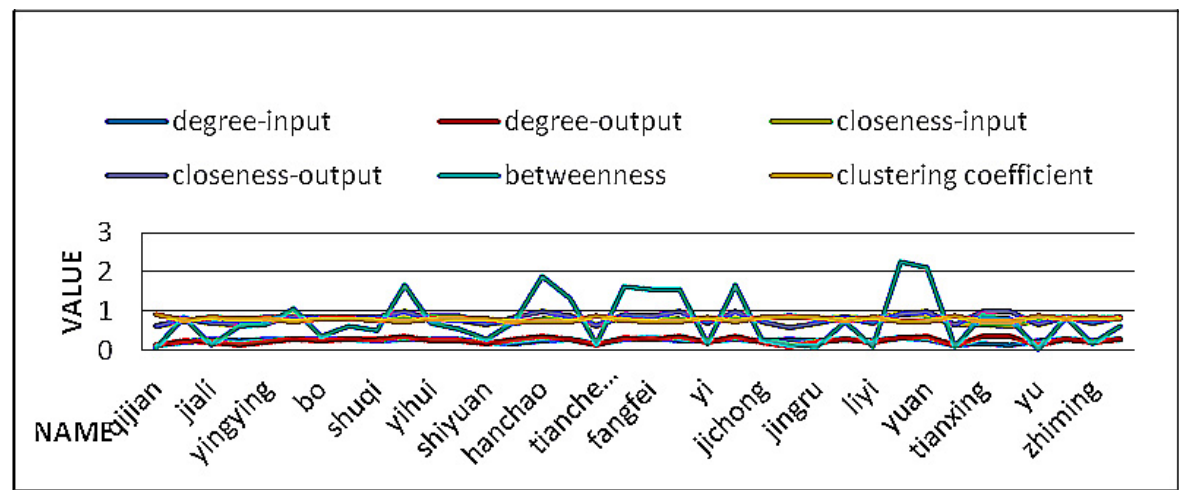

Fig. (7). The comprehensive comparison line chart of topological property.

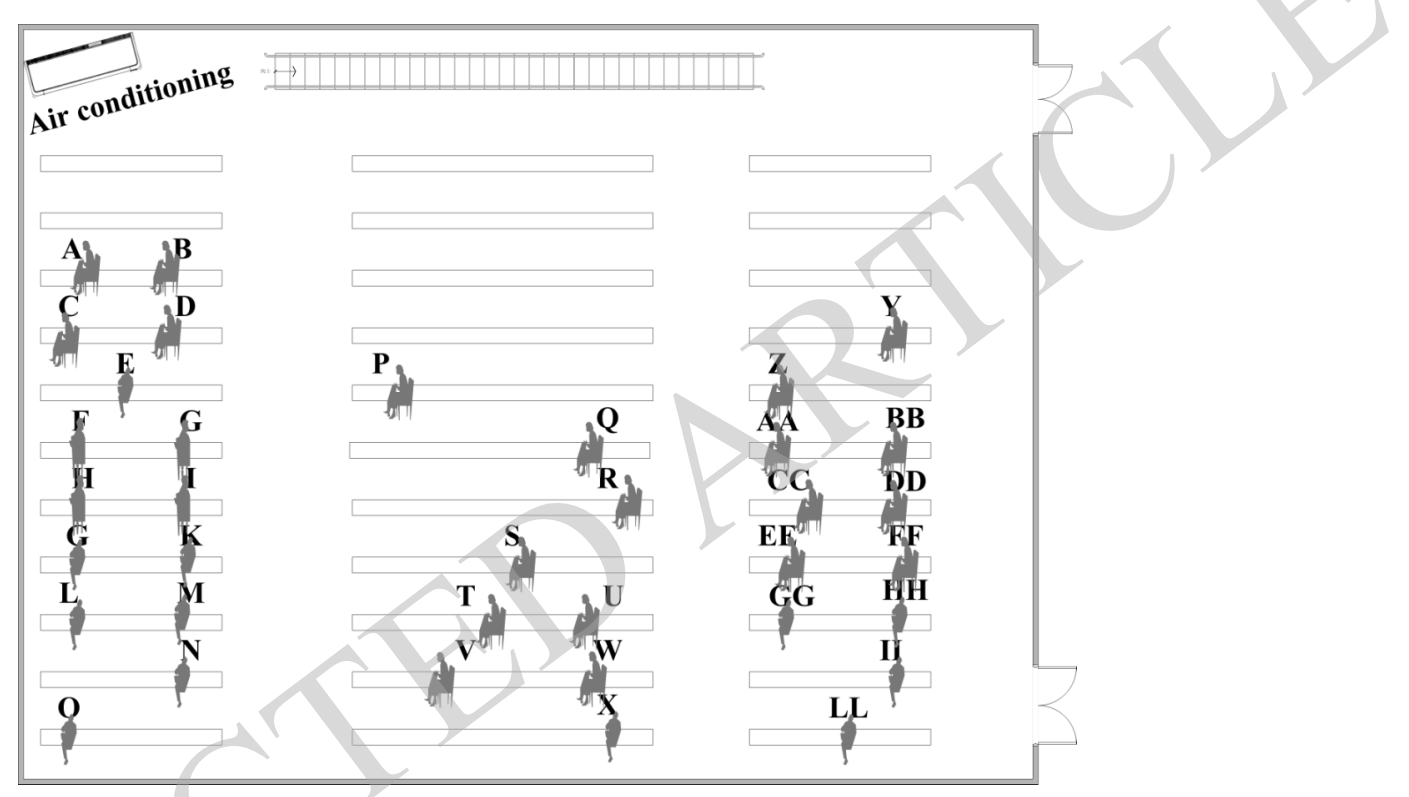

Fig. (8). The seating chart of the students' network.

\section{THE TRAVERSAL OF THE STUDENT'S NET- WORK}

Traversing a network refers to from the beginning node, spread by some rules to affect others from the starting node, gradually, and this effect will be increased, until all the nodes are affected. This process is clearly bound to time factor, and in previous studies on social networks, due to the timeliness of the dissemination of information, convenience and diversity, it is appropriate to ignore the impact of space factors. However, this assumption has limitations. The effects of spatial factors under certain conditions cannot be ignored.

When ignoring the spatial factors, the article is comparing the student network spread sequence with some influence of student network in the actual situation to verify the necessity of considering space factors in certain simulation. The student's network traversal spread is operated by Matlab. The following Fig. (7) is the seating chart of the student's network at night lesson and Fig. (8) shows the students network traversal process in the seating charts, the arrow direction is the direction of propagation:

In order to more clearly observed differences in traversal process, the following Figs. (9) and (10) are pprovided.
Fig. (9) and (10) show the traversal process under the condition of no considering spatial factor only other factors, you can see clearly that it has a big difference with actual situation, because the traversal process only consider the effect of the degree of intimacy between classmates.

Now let's see if spatial factor influence traversal time on students network. First, as shown in Fig. (12), when considering spatial factor, the traversal time is longer, for the space limit the traverse process.

Second, our will study the relationship between traversal time and topological characteristic values. As Fig. (11) shows, network node degrees, betweenness centrality and kcore have no obvious mathematical relationship between the traversal time, but also shown in Fig. (12), at most of the nodes, network closeness centrality have a reverse relationship between traversal time, that is to say, at the big closeness centrality node, the traversal time is shorter than the small nodes, this is because the concept of closeness is used to describe the distance between the node and other nodes in the network. The big closeness means small distance from the node to other nodes. 


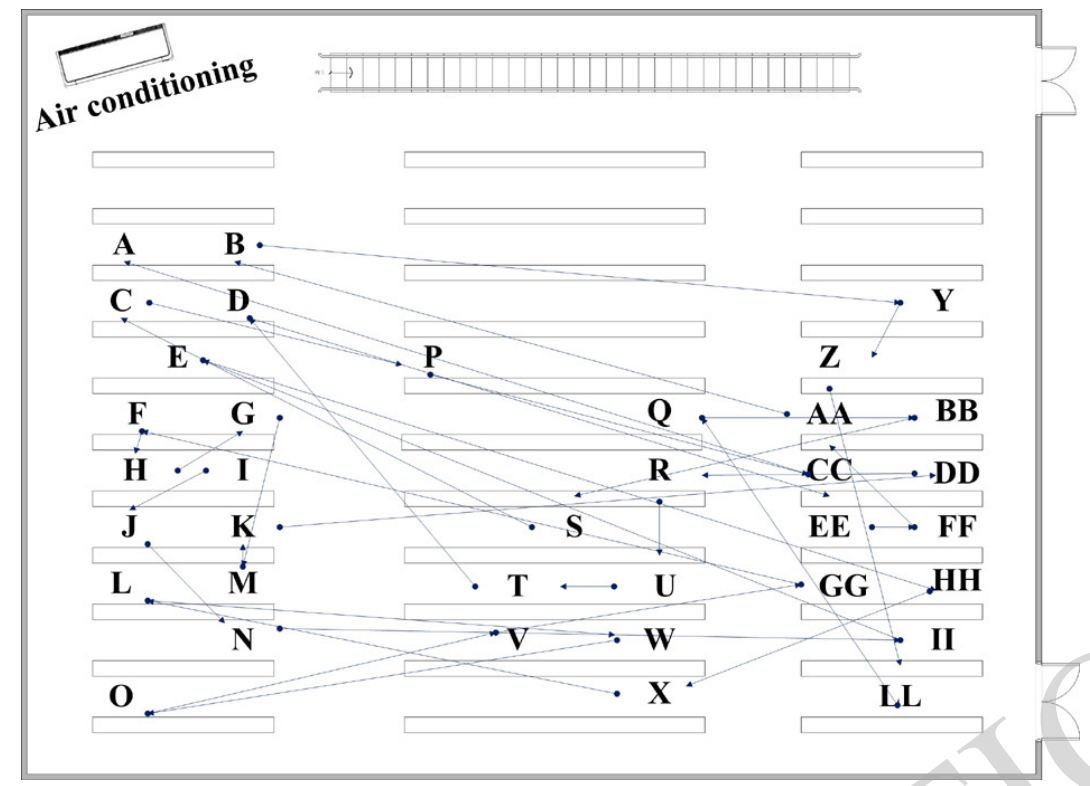

Fig. (9). The result of traverse with random node.

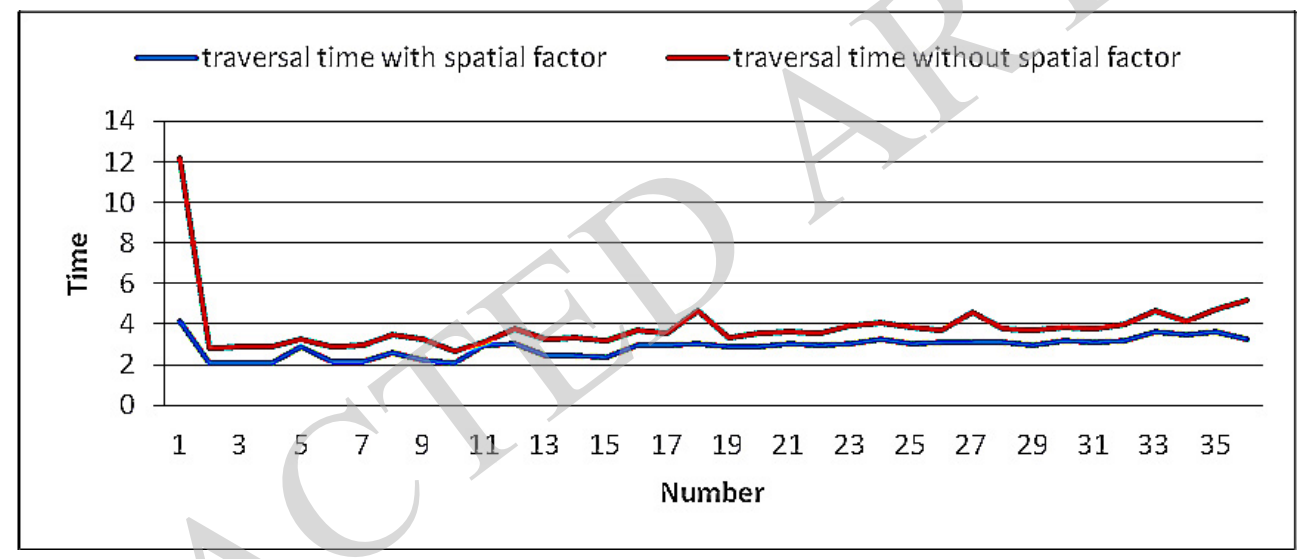

Fig. (10). The comparison diagram of traversal time.

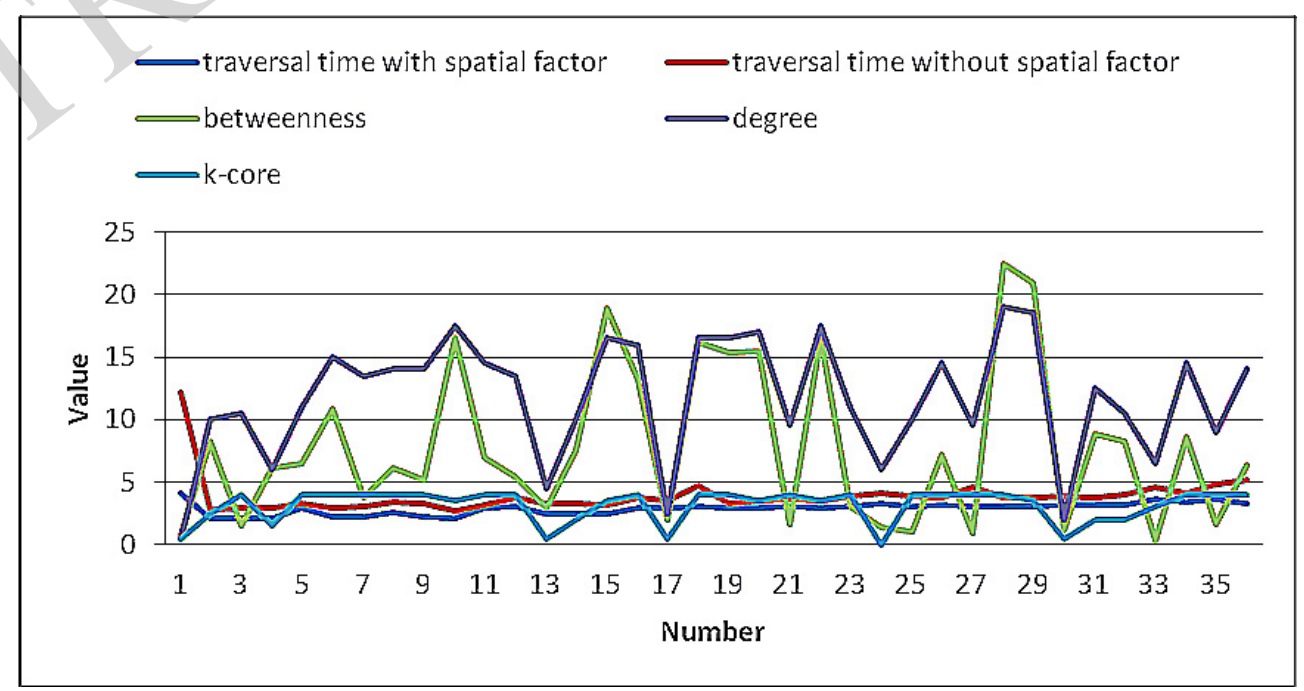

Fig. (11). The relation diagram of other topological property values and traversal time. 


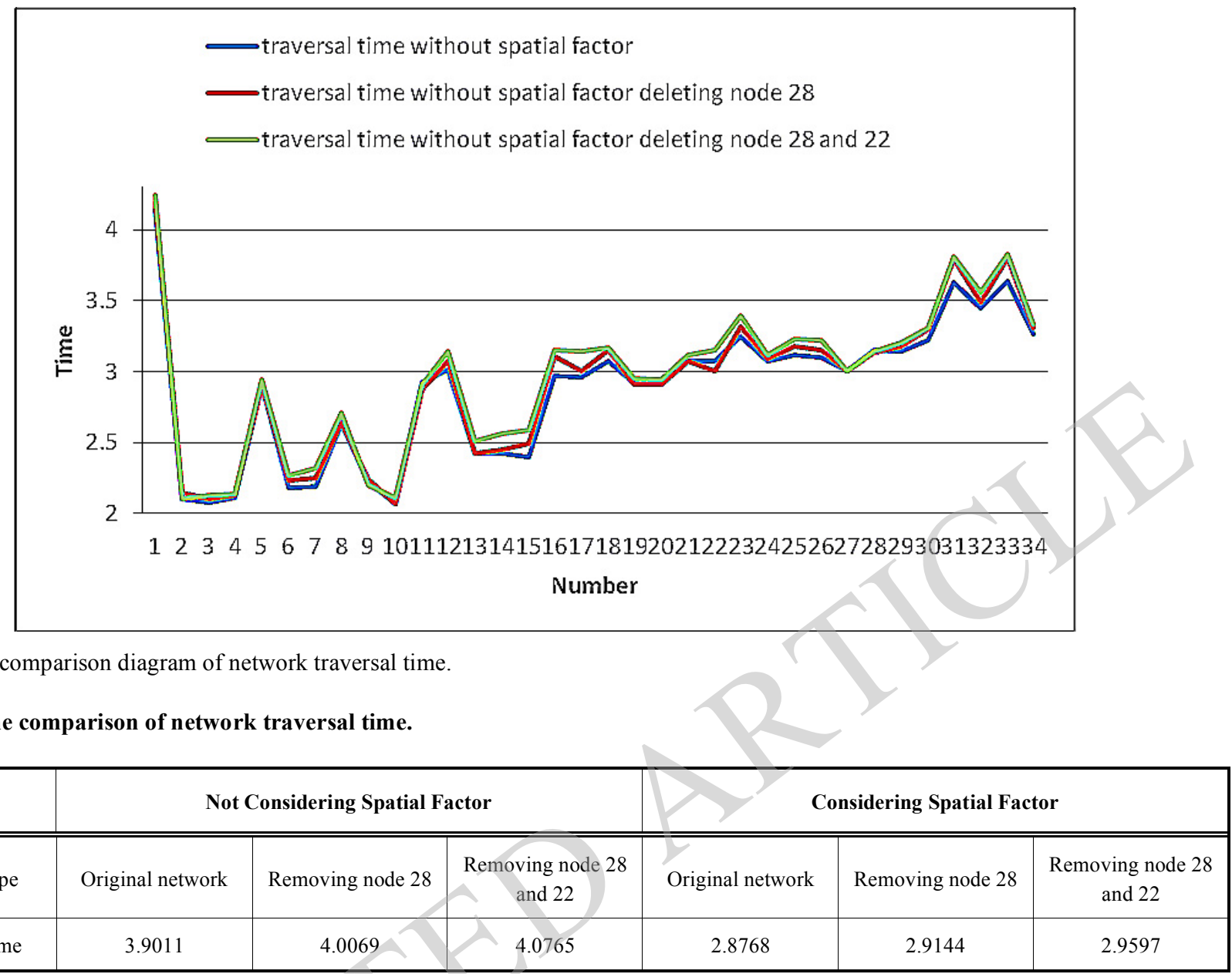

Fig. (12). The comparison diagram of network traversal time.

Table 1. The comparison of network traversal time.

\begin{tabular}{|c|c|c|c|c|c|c|}
\hline & \multicolumn{3}{|c|}{ Not Considering Spatial Factor } & & \multicolumn{2}{c|}{ Considering Spatial Factor } \\
\hline \hline Network type & Original network & Removing node 28 & $\begin{array}{c}\text { Removing node 28 } \\
\text { and 22 }\end{array}$ & Original network & $\begin{array}{c}\text { Removing node 28 } \\
\text { Removing node 28 } \\
\text { and 22 }\end{array}$ \\
\hline Traversal time & 3.9011 & 4.0069 & 4.0765 & 2.8768 & 2.9144 & 2.9597 \\
\hline
\end{tabular}

As shown in Fig. (12), removing the biggest closeness node, like node 28 and 22 , network traversal time is longer than the original, but not particularly clear, this is because the topological characteristics of the students network itself, it is a network of intensive, so remove individual point have little effects on the traversal of the network, but when we make comparisons on average traversal time among several kinds of network, gap can be found, as shown in Table 1.

\section{CURRENT \& FUTURE DEVELOPMENTS}

It is clearly seen from comparison between the above figures and table, when the spatial factor is not considered, there exists a difference between the traversal process and the actual student network traversal process. Because there is no consideration about the impact of spatial factor to weaken the role of the key students, if two students get far away from each other, the status of the other student will be relatively weak, even though one of the students does not learn or does learn. Therefore, from this research, we can get the following conclusion:

1. It is significant to consider the effect of the existence of the space factor when studying the social network, in particular in the simulation process.

2. In numerous topology characteristic parameters, the role of the closeness centrality is more important in the network traversal process.

\section{CONFLICT OF INTEREST}

The authors confirm that this article content has no conflict of interest.

\section{ACKNOWLEDGEMENTS}

This work was supported by Science and Technology Key Research of Beijing Educational Commission (No. KZ201210038038), 2013 State Administration of Work Safety and the National Natural Science Funds (No. $71471121)$.

\section{REFERENCES}

[1] X. Pan, G. Deng, B. Dong, "Based on propagation model building and analysis of social public opinion network", Operat. Manag., vol.55, pp. 176-179, 2011.

[2] Y. Liu, W. Niu, "Mechanism modeling analysis of formation and evolution", Impact Sci. Soc., vol. 46, pp. 10-14, 2009.

[3] M. C. Gonz, A. O. Sousa, H. J. Herrmann, "Opinion formation on a deterministic pseudo-fractal network", Int. J. Mod. Phy. C, vol. 15, pp. 45-57, 2004.

[4] D. Stauffer, "Monte Carlo simulations of Sznajd models", J. Artif. Soc. Soc. Simulat., vol. 5, no. 1, Feb 2003, [Available from: $\mathrm{http}: / /$ jasss.soc.surrey.ac.uk/5/1/4.html]

[5] A. Giladi, "Authenticated encryption support in iso/iec", W. O. Patent 058971, Apr 17, 2014.

[6] W. Hong, "Power saving in cellular networks", W. O. Patent 056174, Apr 17, 2014.

[7] O. Stanze, "Altruistic scheduling in heterogeneous wireless networks", W. O. Patent 0566650, Apr 17, 2014. 
[8] A. Kikuchi, "Communication apparatus", W. O. Patent 057610, Apr 17, 2014.

[9] P. Soon-Shiong, "Distributed storage systems and methods", W. O. Patent 058842, Apr 17, 2014.

[10] N. Shi, "Nodes, systems and methods in a cellular network", W. O. Patent 058379, Apr 17, 2014.

[11] F. G. Radmacher, W. Thomas, "A game theoretic approach to the analysis of dynamic networks", Electron Notes Theor Comput Sci, vol. 200, pp. 21-37, 2008.

[12] Z. Wang, Complex network modeling and application research of interlocking directorates, Qingdao, Shandong province, Ocean University of China, 2009.

[13] B. Yang, Structure measure and model research of the complex social network, Shanghai: Shanghai Jiao Tong University, 2007.

Received: September 22, 2014

Revised: November 30, 2014

Accepted: December 02, 2014

(C) Jun et al.; Licensee Bentham Open.

This is an open access article licensed under the terms of the Creative Commons Attribution Non-Commercial License (http://creativecommons.org/licenses/by-nc/3.0/) which permits unrestricted, non-commercial use, distribution and reproduction in any medium, provided the work is properly cited. 